\title{
Drugs and the lung The effect of oxygen on the lung
}

\author{
ALBERT E. CLAIREAUX \\ From the Institute of Child Health, University of London
}

The harmful effect of oxygen in high concentration on the lung has been recognized for many years. Smith (1899) observed the lethal results following the exposure of experimental animals to oxygen at between 3 and 5 atmospheres pressure over a period of 24 hours. He considered that death resulted from pulmonary inflammation. Karsner (1916) and Binger, Faulkner, and Moore (1927) described widespread changes in the lungs of animals exposed to high concentrations of oxygen for 48 hours or more.

Much of the early work was concerned with animals but later necropsy studies showed that similar pulmonary lesions occurred in man (Pratt, 1958). It was evident that oxygen could exert a harmful effect either over a short period at high pressure or over a longer time at atmospheric pressure.

\section{Hyperbaric Oxygen}

Oxygen administered at high pressures has been used for a variety of clinical conditions such as respiratory failure, cyanotic heart disease, ischaemia, cardiovascular surgery, shock, air embolism and coal gas poisoning (Ledingham, 1969). Pressures about 4 atmospheres are not used clinically because of the dangers of toxicity, which can cause rapid derangement of the central nervous system within a matter of minutes if the oxygen overdose is unduly large or the pressure unduly high-over 2.5 atmospheres. Below 2.5 atmospheres the onset of toxic effects is more insidious and the lungs are the main target. Even at 2 atmospheres pressure, however, some deterioration of lung function may be observed soon after oxygen is administered. The high arterial oxygen tension reached-about $1500 \mathrm{mmHg}$ - gives rise to hyperventilation, possibly as a result of high carbon dioxide tension in the brain. Cardiac output falls and peripheral resistance rises. A reduction in blood flow to every organ ensues. As far as the lungs are concerned, vital capacity, compliance and diffusing capacity are all reduced. These changes are reversible, at least initially, and the onset of lung damage can be delayed by interrupted therapy procedures.

Permanent lung damage, both in the human subject and experimental animal, takes the form of gross congestion and fibrinous exudation into the alveoli. The changes are not dissimilar to those resulting from oxygen toxicity at atmospheric pressure but are of earlier onset.

\section{Oxygen at Atmospheric Pressure}

Pulmonary lesions resulting from prolonged exposure to high levels of oxygen tension are well documented and the term 'respirator lung syndrome' has been coined by Nash, Blennerhassett, and Pontoppidan (1967). These workers found consistent pulmonary damage in patients who had received oxygen therapy and artificial ventilation. These patients showed a progressive deterioration of pulmonary function with reduced compliance and lowered vital capacity. They finally died from pulmonary insufficiency. The investigations of Nash and his colleagues revealed that the lung changes resulted from exposure to oxygen rather than to the effects of artificial ventilation. At necropsy the lungs from their patients were unduly heavy, markedly congested and inelastic and of increased consistency. The cut surface was fleshy in appearance. The histological findings depended upon the duration of the oxygen therapy. In the early stages the microscopic changes were mainly exudative in character. The lungs were very congested and patchy intraalveolar haemorrhage was seen. This was accompanied by fibrinous exudation into the alveoli and hyaline membrane formation. In patients who died after more prolonged exposure to oxygen, the changes were proliferative in character. The interalveolar septa were thickened and fibroblastic proliferation and collagen deposition were observed. A few lymphocytes were seen but no evidence of infection was seen. At the same time diffuse hyperplasia of alveolar lining cells was noted, and was occasionally so pronounced that a cuboidal epithelial layer was seen lining groups of alveoli. According 
to these observers the severity of the lesions was entirely dependent upon the concentration of oxygen and the duration of the exposure to this form of therapy.

The findings of Nash et al (1967) concerned the results of oxygen therapy in 70 adults. They were similar to those reported by Cederberg, Hallsten, and Miörner (1965). Almost precisely similar results were noted in the case reported by Fuson, Saltzman, Smith, Whalen, Osterhout, and Parker (1965) where a girl of 17 years of age was treated by hyperbaric oxygen at a concentration of $100 \%$ at pressures up to 3 atmospheres. Death occurred after five days and here again pulmonary congestion, intraalveolar haemorrhage and hyaline membrane formation were in evidence. The pulmonary lesions are thus almost identical whether hyperbaric oxygen or oxygen at normal atmospheric pressure is used.

These reports all referred to adult patients but recently there have been similar accounts of pulmonary lesions resulting from oxygen therapy in infants and young children. Burrows and Edwards (1970) recorded seven patients whose ages ranged from a few hours to 3 years at the time of onset of oxygen therapy. Six of the patients died. The lungs were heavy and solid. On microscopic examination hyaline membranes were found together with areas of haemorrhage and thickening of alveolar walls. Some alveolar epithelial cell proliferation was also seen. Although hyaline membrane formation is a common accompaniment of the respiratory distress syndrome in the newborn infant, none of these children had suffered respiratory distress at the time of birth and only the youngest patient had become ill during the first day of life. A larger study undertaken by Banerjee, Girling, and Wigglesworth (1972) concerned 81 babies who had received oxygen therapy and who had lived for at least 48 hours. Twenty-three of these infants showed pulmonary lesions at necropsy. The extent of the pulmonary damage could be correlated with the degree of oxygen concentration and the duration of the therapy. Pulmonary fibroplasia was present in all infants who had received over $60 \%$ oxygen for more than 123 hours or over $80 \%$ oxygen for more than 105 hours. At necropsy the lungs of the affected infants were bulky, and on histological examination showed squamous metaplasia of the bronchial epithelium, fibroblastic proliferation and an increase in connective tissue and proliferation of alveolar lining cells. In addition hyaline membrane formation was a prominent feature as was an increase in the musculature and elastic tissue in the walls of terminal bronchioles. It was found that those infants who had been treated with oxygen in concentrations below $60 \%$ did not develop pulmonary fibroplasia.
Although Banerjee et al found it difficult to distinguish between the effects of prolonged oxygen therapy and prolonged artificial ventilation, they did point out that prolonged oxygen therapy at high concentrations always resulted in pulmonary fibroplasia whereas prolonged ventilation did not. However, Barnes, Glover, Hull, and Milner (1969) have shown that infants who require artificial ventilation over a period of six months or more do have impaired pulmonary function even if the oxygen concentration is $50 \%$ or less.

The relationships between hyaline membrane disease and the exudative phase of oxygen toxicity is difficult to define in the newborn infant. Infants with respiratory distress and hyaline membrane formation are among those most likely to require oxygen therapy in the neonatal period and the latter is likely to aggravate a lesion already present. In the study reported by Banerjee et al (1972) two infants with a gestational age of over 41 weeks had pulmonary fibroplasia and well formed membranes. This type of lesion is extremely rare in such infants not exposed to oxygen therapy.

Although most of the studies of pulmonary fibroplasia or ventilator lung in both adults and infants have been concerned with necropsy material, it must be assumed that some patients with similar pulmonary lesions do survive but probably have permanently impaired pulmonary function. In addition it must be suspected that the anatomicak lesions in infants' lungs will interfere with subsequent lung growth, particularly that associated with alveolar development. It is also possible that some air passages will remain blocked by inspissated secretion (Northway, Rosen, and Porter, 1967) and this in turn could cause overdistension of those alveoli still capable of some function and a centrilobular emphysema would develop (Rosan and Lauweryns, 1970).

The pulmonary lesions which result from oxygen toxicity have been reproduced in a variety of experimental animals (Binger et al, 1927)-in rabbits (Karsner, 1916) and more recently in the monkey (Robinson, Harper, Thomas, and Kaplan, 1967). Gerschman, Gilbert, and Caccamise (1958) exposed mice to oxygen at pressures up to 10 atmospheres. The survival time decreased rapidly with increase in pressure and was only 19 minutes at 10 atmospheres. Cedergren, Gyllensten, and Wersäll (1959), also using mice exposed to high concentrations of oxygen, studied the effects produced on the lung ultrastructure. These included injuries to the alveolar walls and exudation between basement membrane and adjacent cells. The effect on guineapig lungs of high concentrations was studied by Bruns and Shields (1954) and by Aikawa and Bruns 


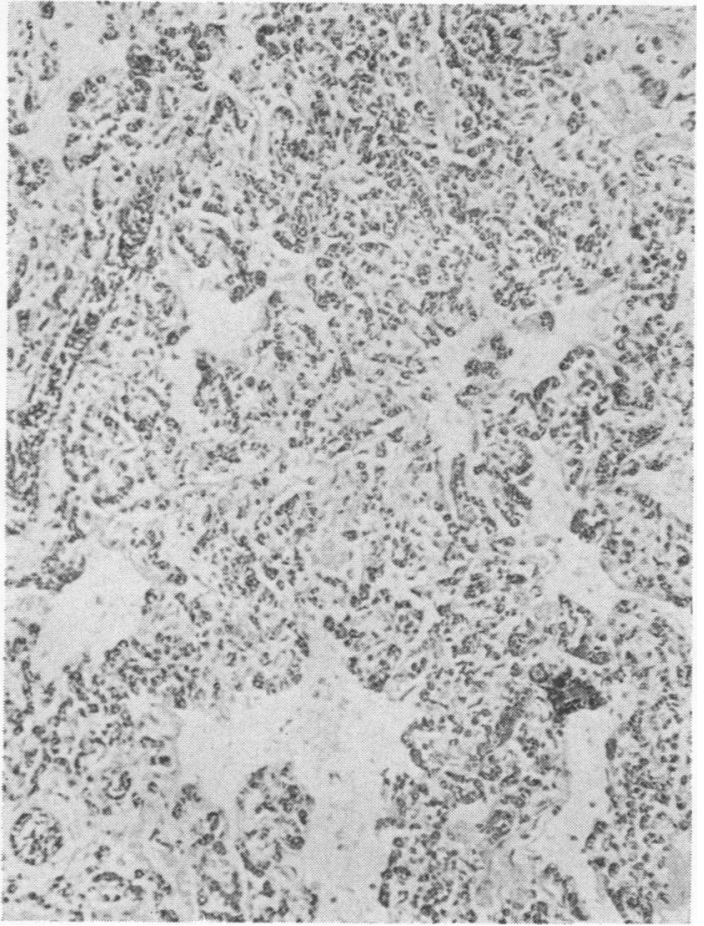

Fig 1

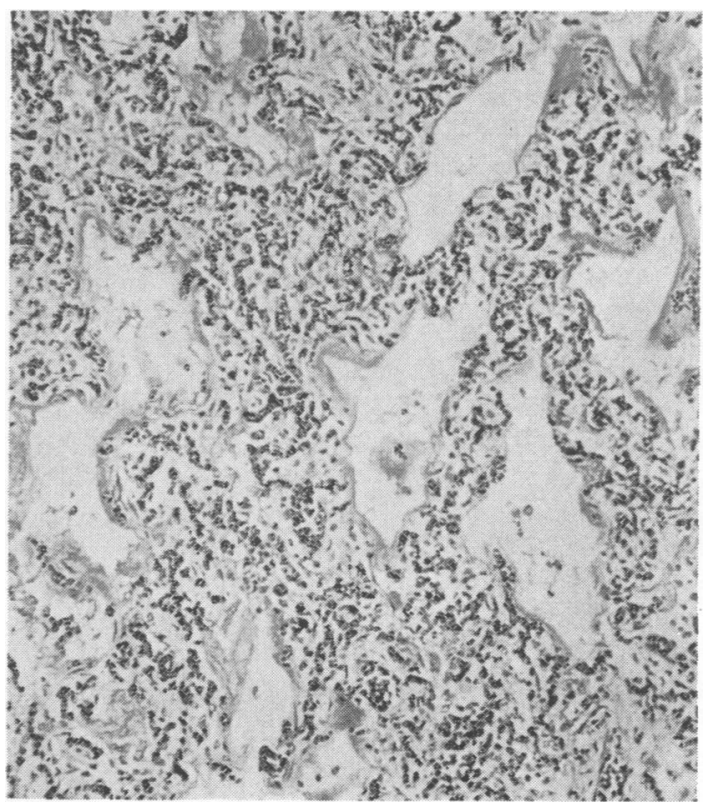

Fig 2

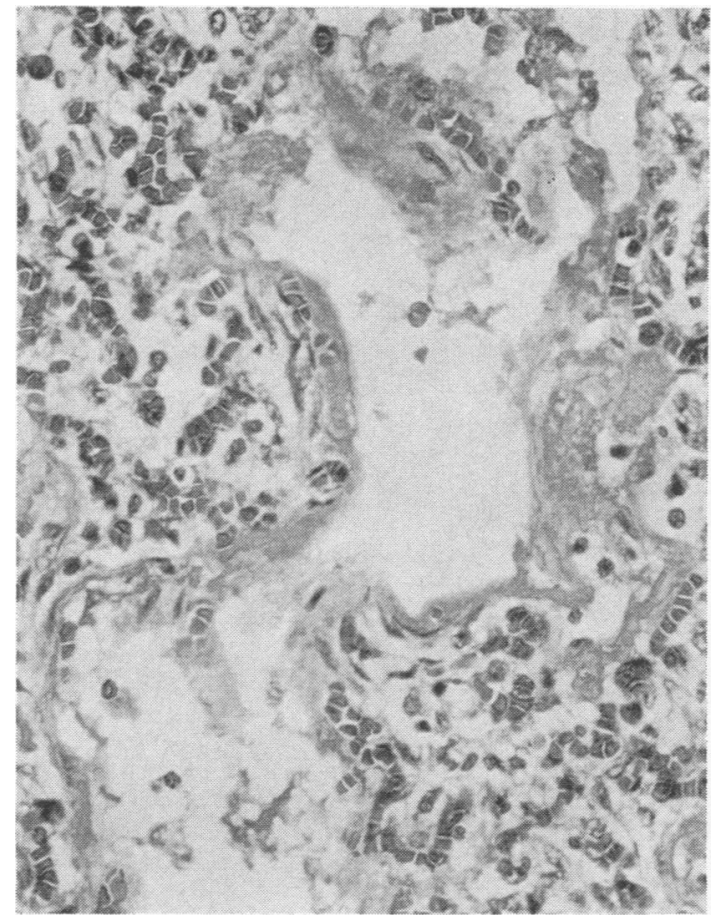

Fig 3

Fig 1 Lung: acute exudative phase. Gross congestion and alveolar exudate. $H \& E \times 75$.

Fig 2 Lung: acute exudative phase. Layering of fibrinous material to form hyaline membranes. $H \& E \times 75$.

Fig 3 Lung: acute exudative phase. Hyaline membrane formation. $H \& E \times 225$. 


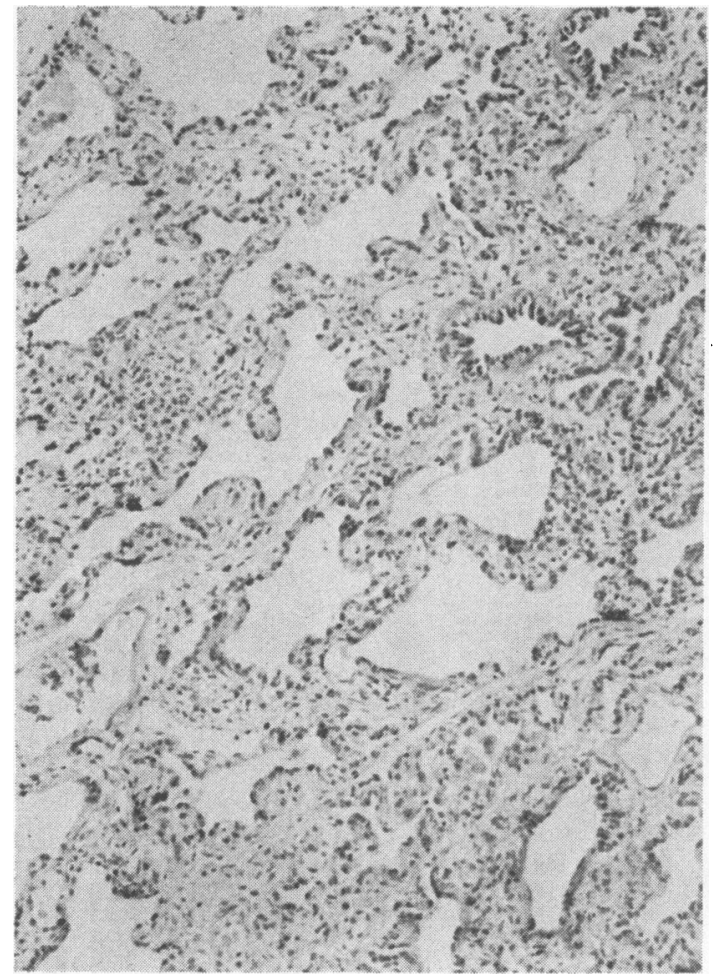

Fig 4

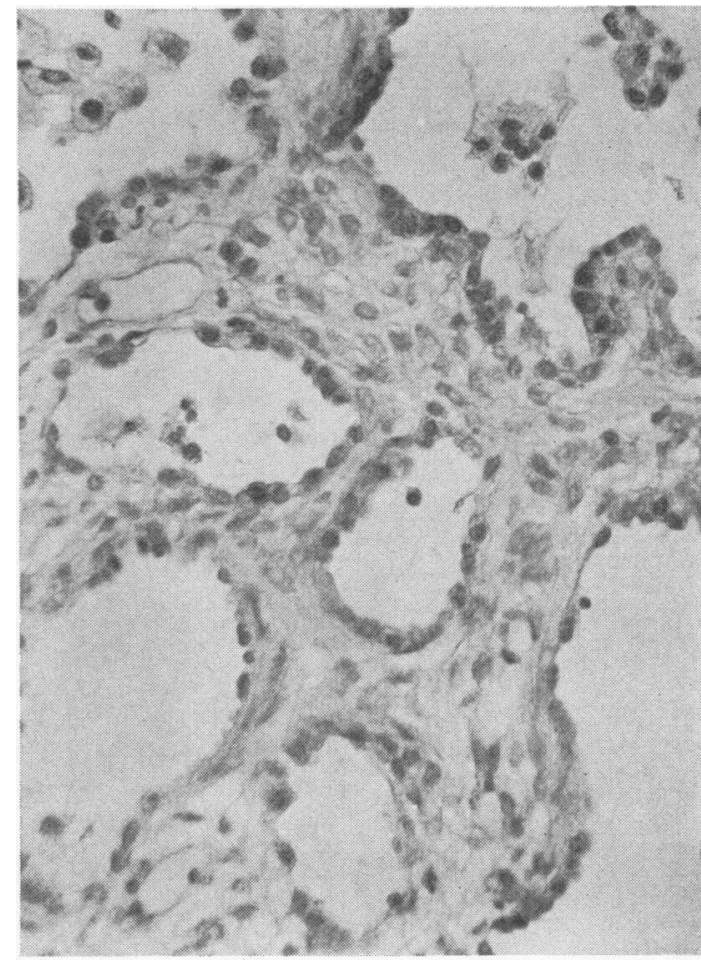

Fig 5

Fig 4 Lung: proliferative phase. Thickening of alveolar septa and proliferation of alveolar lining cells. H\&E $\times 75$.

Fig 5 Lung: proliferative phase. Prominent alveolar lining cells. $H \& E \times 225$.

(1956). These authors produced well formed hyaline membranes in animals exposed to $96 \%$ oxygen for periods of 32 to 64 hours. They also showed that the lesion could be reversible.

Two sets of experiments throw an interesting light upon variables other than the concentration of oxygen involved. The first of these is temperature. Faulkner and Binger (1927) showed that the exposure of cold-blooded animals, eg, the frog or the turtle, to 80 to $90 \%$ oxygen at room temperature over a period of 21 days was without effect. When the oxygen temperature was raised to $37.5^{\circ} \mathrm{C}$, however, the animals died with typical haemorrhagic consolidation of the lungs. The second variable is pressure. Dines and Hiatt (1964) showed that rats were capable of surviving normally for 24 days in $100 \%$ oxygen at subatmospheric pressure.

The critical effect of the pressure at which the animal is exposed to high concentrations of oxygen was also noted by Robinson et al (1967). Their experiments were carried out on monkeys ( $M$. mulatta). They were able to confirm the results of those who had used larger mammals and found the typical pulmonary lesions which were either acute exudative or subacute proliferative in type. However, the most severe and extensive lesions were seen in monkeys exposed at $760 \mathrm{~mm} \mathrm{Hg}$ and those in monkeys exposed at $600 \mathrm{~mm} \mathrm{Hg}$ were mild and focal in distribution.

It is clear from both necropsy studies in man and from various experiments on animals, including primates, that the toxic effect of oxygen on the lung produces severe lesions. These are: (a) acute exudative lesions with massive alveolar oedema, patchy haemorrhage and layering of fibrinous material around alveolar walls to form hyaline membranes (figs 1-3) and (b) proliferative lesions which appear after more prolonged exposure and take the form of proliferation of interstitial tissue, hyperplasia of the alveolar lining cells and desquamation of cells lining the bronchioles and some alveoli (figs 4-6).

The mechanism by which oxygen achieves these 


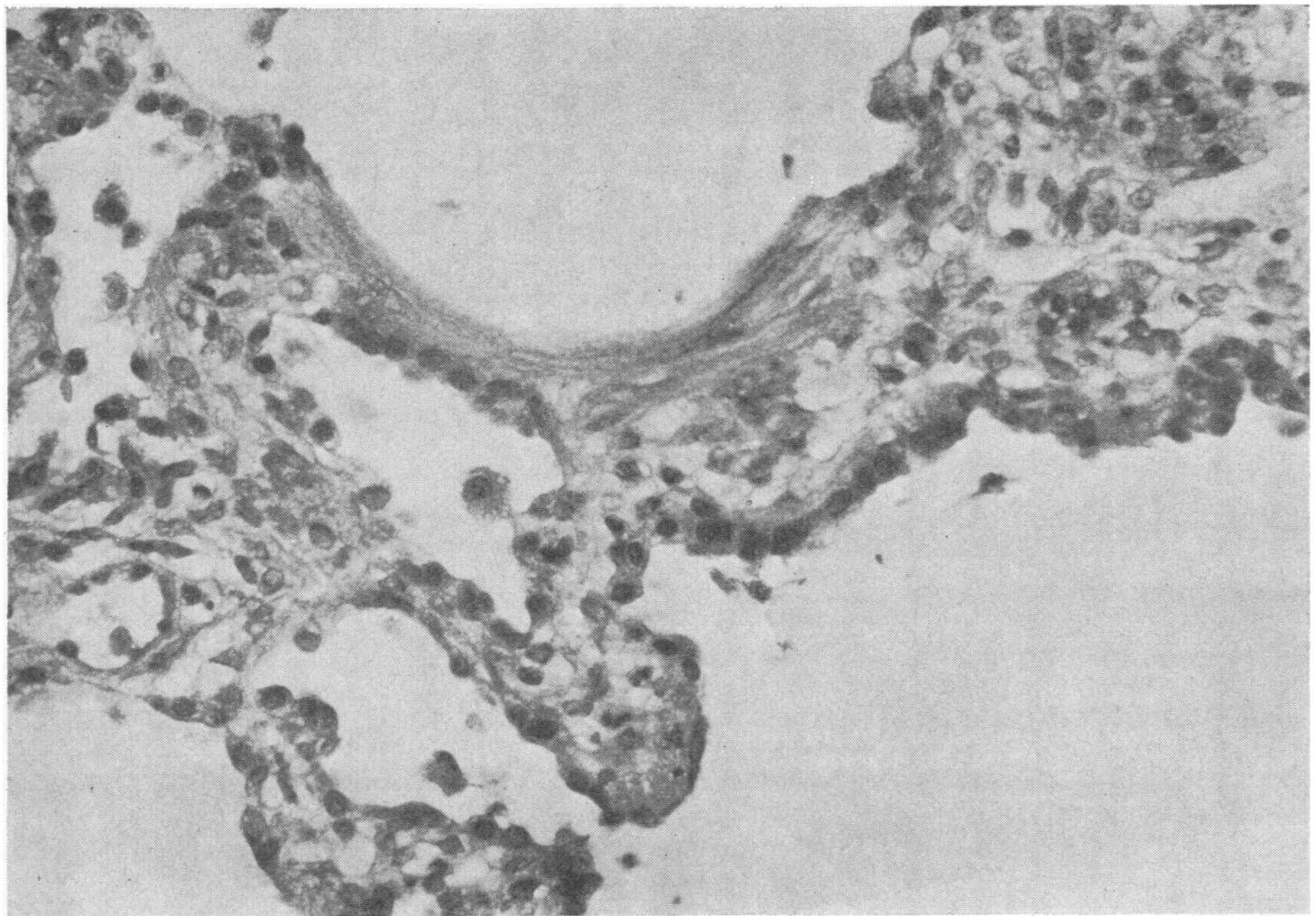

Fig 6 Lung: proliferative phase. Interstitial fibrosis. $H \& E \times 100$.

effects is still unclear. The investigations of Cedergren et al (1959) and others would appear to indicate that there is an increased capillary permeability. Heppleston and Simnett (1964) found that pulmonary alveolar epithelium in tissue culture was especially vulnerable to the effect of oxygen. These workers suggest that oxygen exerts its influence through inhibition of enzyme activity, especially with enzymes containing sulphydryl groups.

The inhibitory effect on sulphydryl-dependent enzymes as a result of oxygen toxicity was also noted by Ludwin, Northway, and Bensch (1974). They investigated the ultrastructure of bronchioles in newborn mice after up to a week's exposure to $100 \%$ oxygen. Other experimental studies had largely concentrated upon pulmonary changes at an alveolar level but Ludwin et al (1974) were able to show that cell death and desquamation of the bronchiolar epithelium took place. They believed that the inhibition of sulphydryl dependent enzymes by oxygen in turn led to a depression of oxidative enzymes. This was reflected morphologically in changes in the mitochondria such as swelling and fragmentation of cristae. Lipid peroxidation also resulted from high oxygen concentration and caused profound membrane damage in cells lining bronchioles such as bleb formation, shortening of cilia and loss of microvilli.

Another pulmonary disorder in man where the aetiology is obscure is the Hamman-Rich syndrome (Hamman and Rich, 1944). In its earlier stages this condition is associated with an acute exudative lesion in the lungs together with pronounced hyaline membrane formation. In the later stages of the disease there is proliferative change and interstitial fibrosis. As Robinson et al (1967) have remarked, the disease bears a very close resemblance to the changes in monkey lung following the administration of oxygen in high concentration at normal atmospheric pressure and the latter might prove a useful model for the study of diffuse interstitial pulmonary fibrosis.

Further studies of a more long-term nature are also proposed by Ludwin et al (1974). They have shown that after seven days' exposure to $100 \%$ oxygen some regeneration of cuboidal cells is taking 
place in the bronchiolar epithelium. At the same time there is an increase in fibroblasts and collagen in the subepithelial connective tissue. This would appear to indicate that not only the lining epithelium but the entire bronchiolar wall is involved in the process of regeneration. These workers are undertaking further long-term experiments to elucidate the factors involved in repair. Hopefully, this will improve our understanding of the nature of epithelial metaplasia and interstitial fibrosis which occur not only as a result of oxygen toxicity but in some other disorders whose aetiology remains obscure.

\section{References}

Aikawa, J. K., and Bruns, P. D. (1956). Pulmonary lesions in experimental oxygen poisoning. Amer. J. Dis. Child., 91, 614-620.

Banerjee, C. K., Girling, D. J., and Wigglesworth, J. S. (1972) Pulmonary fibroplasia in newborn babies treated with oxygen and artificial ventilation. Arch. Dis. Childh., 47, 509-518.

Barnes, N. D., Glover, W. J., Hull, D., and Milner, A. D. (1969). Effects of prolonged positive pressure ventilation in infancy. Lancet, 2, 1096-1099.

Binger, C. A. L., Faulkner, J. M., and Moore, R. L. (1927). Oxygen poisoning in mammals. $J$. exp. Med., 45, 849-864.

Bruns, P. D., and Shields, L. V. (1954). High oxygen and hyaline-like membranes. Amer. J. Obstet. Gynec., 67, 1224-1236.

Burrows, F. G. O., and Edwards, J. M. (1970). A pulmonary disease in patients ventilated with high oxygen concentrations. Brit. $J$. Radiol., 43, 848-855.

Cederberg, A., Hellsten, S., and Miörner, G. (1965). Oxygen treatment and pulmonary hyaline membranes in adults. Acta path. microbiol. scand., 64, 450-458.

Cedergren, B., Gyllensten, L., and Wersäll, J. (1959). Pulmonary damage caused by oxygen poisoning. Acta paediat. (Uppsala), 48, 477-494.

Dines, J. H., and Hiatt, E. P. (1964). Prolonged exposure of young rats to an oxygen atmosphere at reduced pressure. J. appl. Physiol., 19, 17-20.

Faulkner, J. M., and Binger, C. A. L. (1927). Oxygen poisoning in cold blooded animals. J. exp. Med., 45, 865-871.

Fuson, R. L., Saltzman, H. A., Smith, W.W., Whalen, R.E., Osterhout, S., and Parker, R. T. (1965). Clinical hyperbaric oxygenation with severe oxygen toxicity. New Engl. J. Med., 273, 415-419.

Gerschman, R., Gilbert, D. C., and Caccamise, D. (1958). Effect of various substances on survival times of mice exposed to different high oxygen tensions. Amer. J. Physiol., 192, 563-571.

Hamman, L., and Rich, A. R. (1944). Acute diffuse interstitial fibrosis of the lungs. Bull. Johns Hopk. Hosp., 74, 177-212.

Heppleston, A. G., and Simnett, J. D. (1964). The tissue reaction to hyperbaric oxygen. Lancet, $1,1135-1137$.

Karsner, H. T. (1916). The pathological effects of atmospheres rich in oxygen. J. exp. Med., 23, 149-170.

Ledingham, I. McA. (1969). Hyperbaric oxygen. In Recent Advances in Surgery, 7th ed, edited by Taylor. Churchill, London.

Ludwin, S. K., Northway, W. H. Jr., and Bensch, K. G. (1974). Oxygen toxicity in the newborn. Lab. Invest., 31, 425-435.

Nash, G., Blennerhassett, J. B., and Pontoppidan, H. (1967). Pulmonary lesions associated with oxygen therapy and artificial ventilation. New Engl. J. Med., 276, 368-374.

Northway, W. H., Jr., Rosan, R. C., and Porter, D. Y. (1967). Pulmonary disease following respirator therapy of hyaline membrane disease; bronchopulmonary dysplasia. New Engl. J. Med., 276, 357-368.

Pratt, P. C. (1958). Pulmonary capillary proliferation induced by oxygen inhalation. Amer. J. Path., 34, 1033-1049.

Robinson, F. R., Harper, D. T. Jr., Thomas, A. A., and Kaplan, H. P (1967). Proliferative pulmonary lesions in monkeys exposed to high concentrations of oxygen. Aerospace Med., 38, 481-486.

Rosan, R. C., and Lauweryns, J. M. (1970). Clinicopathological aspects of oxygen toxicity of the newborn human and animal (bronchopulmonary dysplasia). J. Path., 101, pp. xiii-xiv.

Smith, J. L. (1899). The pathological effects due to increase of oxygen tension in the air breathed. J. Physiol. (Lond.), 24, 19-35. 